\title{
A numerical micro-mechanical study of the influence of fiber-matrix interphase failure on carbon/epoxy material properties
}

\author{
Danzi F., Fanteria D., Panettieri E., Palermo M. \\ Università di Pisa \\ Dipartimento di Ingegneria Civile e Industriale \\ Via Girolamo Caruso, 8 - I-56122, Pisa, Italy
}

\begin{abstract}
A finite element micromechanical study of unidirectional carbon-epoxy system is performed in order to investigate the role of fiber-matrix debonding in the degradation of mechanical properties and in the onset of failure for this class of composite materials.

The presence of interphase flaws, that can be induced during the manufacturing processes, into micro-scale FE models is obtained by means of an original damage injection technique developed by the authors. The fibers are considered as transversally isotropic solids and the matrix is modeled as an isotropic, elasto-plastic, material with damage.

The effect of fiber-matrix debonding is analyzed by means of a quasi 3-D unitary cell with a single fiber, with periodic boundary conditions, for different loading cases. Subsequently, multi-fiber representative volume elements are investigated with the same boundary and loading conditions. Finally, the effect of a 3-D debonding propagation is studied via single fiber model with an increased fiber-wise depth.
\end{abstract}


Keywords: Micromechanics, Fiber-Matrix debonding, Polymer matrix composites, Finite element analysis.

\section{Introduction}

The last few years have seen a considerable increase in the use of manufacturing processes for composite structures based on dry fiber pre-forms and different resin injection technologies (Liquid resin infusion, resin transfer moulding and variants). While promising a number of advantages in terms of costs and flexibility of production, such manufacturing technologies are prone to increase the density of micro-defects when compared to conventional prepreg based composited.

Such defects mainly occur in form of voids in the matrix and of decohesions between the matrix and the fibers.

The effect of voids into the polymeric matrix in terms of degradation of the composite mechanical properties has been widely studied $[1,2]$ while the consequences of fiber matrix inter-facial decohesions are still an open issue that is receiving a great attention from the scientific community $[3,4,5,6,7]$. Computational micromechanics on appropriate volumes of material can be advantageously used to understand how interfacial defects may grow and possibly cause matrix damage to initiate and to evolve under different loading scenarios. Homogenization techniques can be then applied in order to calculate the resulting strength of the material from the solution at the micro-scale $[8]$.

In order to have a good model of a fibrous composite at the micro-scale, by which fiber-matrix debonding propagation, matrix damage onset and, 
ultimately, material failure can be studied, the following problems must be addressed:

- Accurately reproducing the distribution of fibers, with representative randomness, in micro-scale models at high fiber volume fractions $[9$, $10,11]$.

- Adequately modeling the mechanical behavior of the constituents $[12$, $3,13,14]$

- Allowing damage onset and propagation into the matrix $[11,15]$.

- Modeling the propagation of fiber-matrix decohesion $[5,6,16,15]$.

- Introducing distributions of discrete fiber-matrix decohesions to represent appropriately possible interfacial defects.

If one want to address the influence of fiber-matrix interphase defects on the mechanical properties of an unidirectional Carbon/Epoxy material, the last point is critical and has not yet been addressed in the literature. The paper presents a possible approach based on user tailored cohesive elements, that model the fiber-matrix interface, whose damage can be initialized through an original technique developed by the authors for meso-scale analyses [17]. A study is presented, based on micro-mechanical analyses, that aims at evaluating the influence of the position, extension, shape and number of defects on the mechanical properties of an UD composite. The analyses are carried out on FE models created, within the commercial software ABAQUS, using python scripting. Through scripting RVE models, with random distributions of fibers, are generated and periodic boundary conditions (PBCs) imposed 
in order to reproduce different loading scenarios (Section 2). The fibers are assumed to behave as a linearly elastic transversely-isotropic material while the matrix is modeled to have a non-linear pressure-dependent elasto-plastic behavior (Section 3).

Constitutive equations for both the matrix and interfaces are defined via user material subroutines (UMAT) while the interface initialization is implemented through a specific subroutine (SDVINI), which defines the initial state-variable field (Section 4).

Since the damage initialization technique has been developed at larger scales, the technique is verified, in the linearly elastic regime, on simple single-fiber models whose results are compared with those of standard FE models in which the fiber is physically detached from the matrix (Section 5).

Low depth fiber-wise models (single fiber and RVE) are created in order to identify the degradation of the transverse properties in presence of quasi 3-D defects, that is long fiber-matrix decohesions characteristic of problematic manufacturing processes (Sections 6 and 7).

Eventually, fiber-wise deep models are developed in section 8 to study the propagation of small, localized, interfacial defects.

\section{Micromechanical model development}

In order to generate micro-mechanical models capable of reproducing the mechanical behavior of an unidirectional composite material many aspects have to been taken into account: an algorithm is developed to reproduce fiber distributions with appropriate spatial statistics within a given volume of material, a micro-scale FE model of such volume is created and specific 
periodic boundary conditions are applied, finally scripts are developed to extract homogenized stress response from the stress field calculated by the FE analyses.

\subsection{The algorithm for fiber distribution generation}

In order to create models with a microstructure as similar as possible to the one of unidirectional carbon/epoxy composites, the authors developed an algorithm for the generation of fiber distributions inspired to the work of Yang and coworkers [10]. The proposed algorithm is able to produce models with square cross section of any size, volume fraction, and fiber clustering. Given a square domain $\mathcal{D}$ of side $l$, the algorithm places the first fiber, of radius $r_{f}$, in a random position inside $\mathcal{D}$, then it starts trying to place fibers in a circular crown sub-domain $\mathcal{C}$ that surround the first fiber, until the subdomain is saturated. Subsequently, the algorithm considers the second fiber it generated as the center for a new circular crown to be filled with fibers. The procedure is repeated until the required fiber volume fraction is reached. Fig. 1 provides a graphical representation of the first step of the algorithm. The

circular crown domain $\mathcal{C}$ is defined by two parameters $r_{\min }$ and $r_{\max }$. The first, $r_{\min }$, identifies the internal radius of the crown and fixes the minimum distance that can exist between two fibers defined as $\Delta_{\min }=r_{\min }-2 r_{f}$; the latter can be defined experimentally by optical analyses of composite microstructures. The maximum radius of the crown, $r_{\max }$, is a function of the required volume fraction and it influences the clustering of the fibers that is typical of the process use to manufacture the composite material. Other values required by the algorithm are the desired volume fraction $\left(V_{f}\right)$, and the size of the cross section specified via the parameter $\delta=l / r_{f}$. 


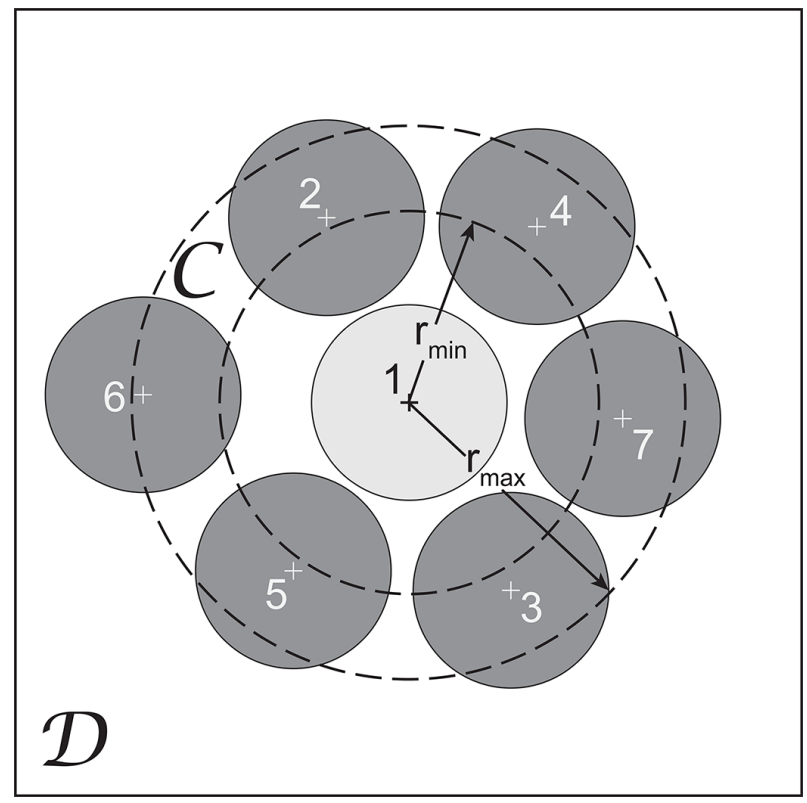

Figure 1: Fiber placement sequence for RVE generation

Fig. 2 shows different distributions of fibers generated at constant crosssection size $(\delta=10)$ and with different volume fraction. We observe that the developed algorithm ensures the condition of material periodicity, which, as shown in the work of Gitman et al.[9], has a great importance for the stability of the global response of micro-mechanical simulations.

\subsection{FE model development and homogenization of the results}

In order to create the 3D FEM models the authors have developed an ABAQUS Python script [18] capable of generating FE models from a given fiber distribution and once the fiber-wise depth of the model is defined. Finite element models are created via an orphan mesh technique with pre- 


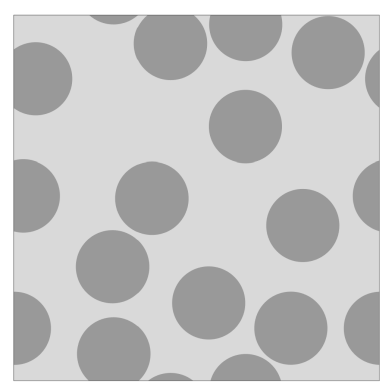

(a) $V_{f}=40 \%$

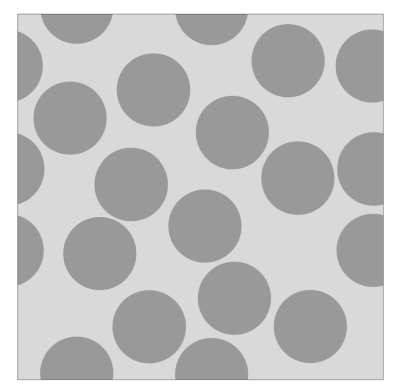

(b) $V_{f}=50 \%$

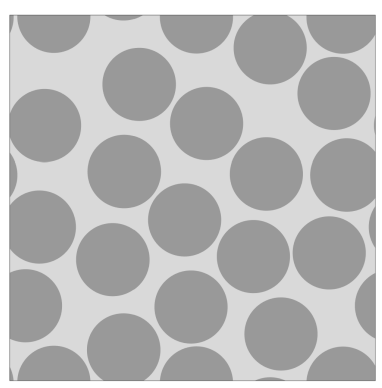

(c) $V_{f}=60 \%$

Figure 2: Examples of fiber distributions for $r_{f}=3.5 \mu m$ at $\delta=10$ and different volume fractions

dominantly first order hexahedral elements (C3D8) ${ }^{1}$ and first order cohesive elements (COH3D8) that model the inter-phase between the fiber and the matrix.

Both solid and cohesive elements have user material properties defined according to constitutive models described in section 3.

Periodic boundary conditions (PBCs) have been applied to the models in order to ensure a macroscopically uniform stress-strain field as described in Barbero [19] and Melro et al. [15].

Stress-strain curves in the different analyses are obtained using an homogenization procedure for the stress according to equation 1 :

$$
\overline{\boldsymbol{\sigma}}=\frac{1}{V} \sum_{P=1}^{N_{P}} V_{P} \boldsymbol{\sigma}\left(\boldsymbol{X}_{\boldsymbol{P}}\right)
$$

where $\overline{\boldsymbol{\sigma}}$ is the homogenized stress tensor, $V$ is the RVE volume, $\boldsymbol{\sigma}\left(\boldsymbol{X}_{\boldsymbol{P}}\right)$ is the stress tensor at the integration point $P$ of coordinates $\boldsymbol{X}_{\boldsymbol{P}}$ and with a

\footnotetext{
${ }^{1}$ Due to the complex geometry a few wedge elements (C3D6) are usually present in the models.
} 


\begin{tabular}{ccccc}
\hline$E_{1}[G p a]$ & $E_{2}[G p a]$ & $G_{12}[G p a]$ & $\nu_{12}[-]$ & $\nu_{23}[-]$ \\
\hline 225. & 15. & 15. & 0.2 & 0.2 \\
\hline
\end{tabular}

Table 1: Carbon fibre elastic properties

relevant volume $V_{P}$.

\section{Advanced modeling of constituent properties}

Once defined the model geometry, another important aspect that must be considered in order to obtain a micro-mechanical model capable of reproducing the physical behavior of the material is the correct definition of the mechanical behavior of the two main constituents: the carbon fibers and the epoxy matrix.

\subsection{Transversally isotropic carbon fibers}

Carbon fibers are assumed to behave as a transversally isotropic linearly elastic solids. The values of the five independent constants that characterize the AS4 carbon fibers considered in the study are taken from [20] and are reported in Table 1.

\subsection{Elasto-plastic damage model for the polymeric matrix}

The epoxy matrix is assumed to be an isotropic elasto-plastic solid with isotropic damage. Consequently, the relation between the stress tensor $(\boldsymbol{\sigma})$ and the elastic strain tensor $\left(\boldsymbol{\epsilon}_{e}\right)$ is defined using the equation proposed by Simo et al. in [21]:

$$
\boldsymbol{\sigma}=(1-d) \mathbf{C}: \boldsymbol{\epsilon}_{e}
$$


For an elasto-plastic solid equation 2 can be specified as:

$$
\boldsymbol{\sigma}=(1-d) \mathbf{C}:\left(\boldsymbol{\epsilon}-\boldsymbol{\epsilon}_{p l}\right)
$$

where $d$ is the damage variable, $\boldsymbol{C}$ is the fourth-order elasticity tensor, $\boldsymbol{\epsilon}$ is the total deformation tensor and $\boldsymbol{\epsilon}_{p l}$ is the plastic deformation tensor.

Since this class of material exhibits a yield behavior that is sensitive to hydrostatic stress as shown by Raghava et al. [22], Fiedler et al. [12] and many other authors, classical Von-Mises or Tresca yield criteria are not adequate. For this reason a modified Von-Mises yield criterion is used with a non associative flow rule as proposed by Melro et al. [11]. The yield condition, in the space of principal stresses, is represented by a paraboloidal; the latter can be conveniently expressed using the stress tensor invariants as:

$$
\Phi\left(\boldsymbol{\sigma}, \sigma_{y c}, \sigma_{y t}\right)=6 J_{2}+2 I_{1}\left(\sigma_{y c}-\sigma_{y t}\right)-\sigma_{y t} \sigma_{y c}=0
$$

where $J_{2}=1 / 2\left(\boldsymbol{\sigma}_{d e v}: \boldsymbol{\sigma}_{\text {dev }}\right)$ is the second invariant of deviatoric stress tensor $\boldsymbol{\sigma}_{d e v}, I_{1}=\operatorname{tr}(\boldsymbol{\sigma})$ is the first invariant of the stress tensor. Symbols $\sigma_{y t}$ and $\sigma_{y c}$ indicate the tensile and compressive yielding stress respectively and are both function of the equivalent plastic strain defined as:

$$
\bar{\epsilon}_{p l}=\sqrt{\frac{1}{1+2 \nu_{p l}^{2}} \boldsymbol{\epsilon}_{p l}: \boldsymbol{\epsilon}_{p l}}
$$

Given that plastic behavior of epoxies can not be captured with an associative model,the following non-associative flow rule is introduced:

$$
g(\boldsymbol{\sigma})=\sigma_{v m}^{2}+\alpha p^{2}
$$

where $\sigma_{v m}=\sqrt{3 J_{2}}$ is the Von-Mises equivalent stress, $p=I_{1} / 3$ is the hydrostatic pressure and $\alpha$ is the material parameter responsible for the correct 
definition of the volumetric component of the plastic flow that is given by:

$$
\alpha=\frac{9}{2} \frac{1-2 \nu_{p l}}{1+\nu_{p l}}
$$

Only isotropic hardening has been taken into account and exponential hardening laws are used in order to reproduce both tensile and compressive behavior of the resin; the shear response is obtained as a result.

A failure surface has been defined that activate the isotropic damage model with an exponential softening law. In order to mitigate the mesh dependency due to softening, the damage model is implemented with a characteristic length correction derived from Bažant crack band theory [23]. The equation that define the damage surface within the space of principal stresses is:

$$
\frac{6 J_{2}}{\sigma_{f t} \sigma_{f c}}+\frac{2 I_{1}\left(\sigma_{f c}-\sigma_{f t}\right)}{\sigma_{f t} \sigma_{f c}}-1=0
$$

The exponential damage evolution law is given in the equation (9):

$$
d=1-\frac{e^{A\left(3-\sqrt{7+2 r^{2}}\right)}}{\sqrt{7+2 r}-2}
$$

where $A$ is the parameter responsible for the energy release rate, correlated with the element size, and $r$ is the damage internal variable. Table 2 summarizes the matrix properties used in the following analyses; they are derived from the experimental results obtained by by Fielder et al. [12].

Symbols in Table 2 are defined as follows: $\sigma_{y t_{0}}$ and $\sigma_{y c_{0}}$ are the tension and compression yield stresses, $\sigma_{f t}$ and $\sigma_{f c}$ are the tension and compression failure stresses, $\nu_{p l}$ is the plastic Poisson's ratio and $G$ is the Mode I fracture toughness. Fig 3 shows the matrix stress-strain curves for uniaxial tension, compression and pure shear, respectively. 


\begin{tabular}{lll}
\hline Property & Value & \\
\hline$E$ & 3760. & {$[M p a]$} \\
$\nu$ & 0.39 & {$[-]$} \\
$\sigma_{y t_{0}}$ & 29. & {$[M p a]$} \\
$\sigma_{f t}$ & 93. & {$[M p a]$} \\
$\sigma_{y c_{0}}$ & 40. & {$[M p a]$} \\
$\sigma_{f c}$ & 159.8 & {$[M p a]$} \\
$\nu_{p l}$ & 0.3 & {$[-]$} \\
$G$ & 0.09 & {$\left[\mathrm{~J} / \mathrm{m}^{2}\right]$} \\
\hline
\end{tabular}

Table 2: Epoxy resin properties

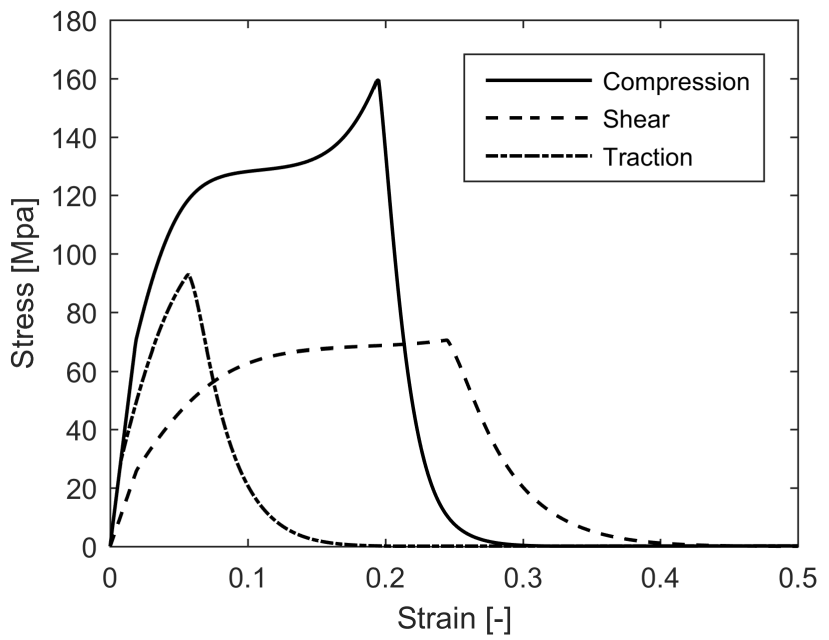

Figure 3: Stress-strain curves for epoxy resin under different loading condition 


\section{Inter-phase modeling}

Key point of the following analyses is the characterization of the fibermatrix inter-phase that include both the capability of make decohesion propagates and the possibility to modify the initial properties of this phase in order reproduce the possible presence of micro-defects induced during the curing process.

\subsection{Cohesive elements for the inter-phase modeling}

In order to satisfy all this requirement and given the small thickness of the inter-phase respect to fiber and matrix dimension the authors propose to model this critical zone with 3-D cohesive elements (COH3D8) governed with a traction separation constitutive behavior. The elements kinematic is managed by ABAQUS while their constitutive equation is governed via an user-material subroutine (UMAT). Thanks to this approach, in fact, is possible both to have a full control over the material behavior and to initialize its state variables according to the technique that will be explained in the following section.

Cohesive elements, assumed with a constant thickness of $0.01 \mu m$, are oriented as reported in Fig. 4 where the normal direction, $n$, is radial from the center of each fiber; the $t_{h}$ shear direction is the hoop direction and $t_{l}$ is longitudinally parallel to the fibre direction.

The main features of the cohesive constitutive model are briefly presented in this section, further details can be found in [24] and [18]. The cohesives 


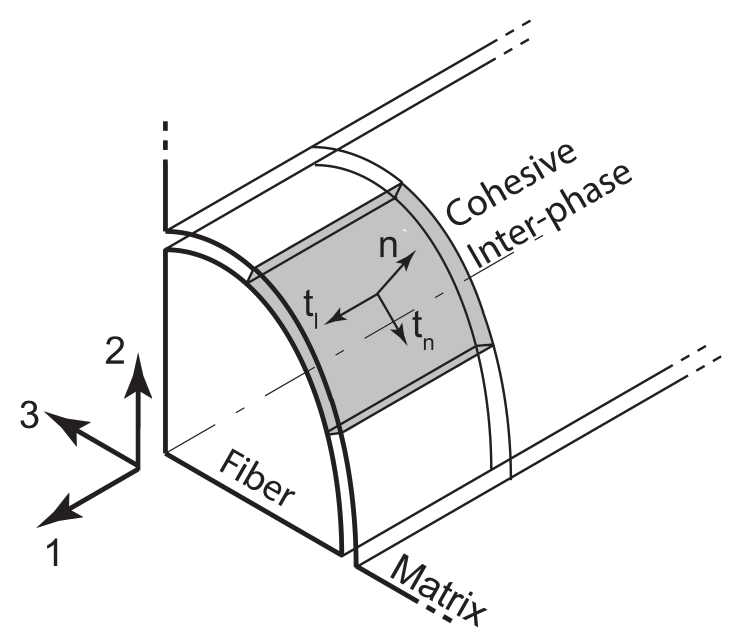

Figure 4: Orientation of cohesive elements

governing equation is given by:

$$
\boldsymbol{\tau}=\left(\begin{array}{c}
\tau_{n} \\
\tau_{s} \\
\tau_{t}
\end{array}\right)=\left(1-d_{i}\right) k\left(\begin{array}{c}
\delta_{n} \\
\delta_{s} \\
\delta_{t}
\end{array}\right)-d_{i} k\left(\begin{array}{c}
<\delta_{n}> \\
0 \\
0
\end{array}\right)
$$

where $\boldsymbol{\tau}$ and $\boldsymbol{\delta}$ are respectively the tension vector and the nodal displacement vector where the subscripts of their components correspond to the three fracture modes: opening $(\mathrm{n})$, shearing $(\mathrm{s})$ and tearing $(\mathrm{t})$ mode while the term into Macauley brackets $\langle$. $\rangle$ is used to neglect negative values of $\delta_{n}$ since contact between debonded surfaces prevents inter-penetration. $k \mathrm{n}$ instead, is the element penalty stiffnesses for the three opening mode and $d_{i}$ is the inter-phase damage variable. Damage onset is predicted by means of a quadratic criterion, with the further assumption of equal shearing and 
tearing strengths (i.e. $\tau_{s}^{0}=\tau_{t}^{0}=\tau_{\text {shear }}^{0}$ ):

$$
\left(\frac{<\tau_{n}>}{\tau_{n}^{0}}\right)^{2}+\left(\frac{\tau_{\text {shear }}}{\tau_{\text {shear }}^{0}}\right)^{2}=1
$$

with $\tau_{\text {shear }}=\sqrt{\left(\tau_{s}\right)^{2}+\left(\tau_{t}\right)^{2}}$. Damage evolution is governed by a linear,energybased, damage evolution law where the mode mixity is taken into account using the BenzeggaghKenane (BK) criterion [25].

$$
G^{c}=G_{n}^{c}+\left(G_{\text {shear }}^{c}-G_{n}^{c}\right)(B)^{\eta}
$$

Where $G^{c}$ is the mixed mode fracture energy that with the subscript $n$ and shear it refers respectively to opening and shearing fracture energy while $\eta$ in the BK exponent and $B$, the mode-mixity ratio, is defined as:

$$
B=\frac{G_{\text {shear }}}{G_{n}+G_{\text {shear }}}
$$

Numerical values used in the following analyses, derived from [16] and [26], are reported in Tab. 3.

\subsection{Inter-phase damage injection technique}

The presence of defects into the fiber-matrix inter-phase is introduce into the models using an ad hoc technique developed by the authors [17]. The proposed method is based on the ABAQUS subroutine SDVINI, which can be invoked before the first increment of the simulations in order to define the initial values of internal state variables (STATEV) present into the user defined material subroutines (UMAT). This approach, developed for the injection of delaminations into flat composite laminates, has been enriched and appropriately modified in order to be applied into more complex domains. 


\begin{tabular}{lll}
\hline Property & Values & \\
\hline$K$ & 500. & {$[\mathrm{Gpa} / \mathrm{mm}]$} \\
$\tau_{n}$ & 85. & {$[\mathrm{Mpa}]$} \\
$\tau_{s}$ & 125. & {$[\mathrm{Mpa}]$} \\
$\tau_{t}$ & 125. & {$[\mathrm{Mpa}]$} \\
$G_{n}$ & 10. & {$\left[\mathrm{~J} / \mathrm{m}^{2}\right]$} \\
$G_{s}$ & 25. & {$\left[\mathrm{~J} / \mathrm{m}^{2}\right]$} \\
$G_{t}$ & 25. & {$\left[\mathrm{~J} / \mathrm{m}^{2}\right]$} \\
$\eta$ & 1.0 & {$[-]$} \\
\hline
\end{tabular}

Table 3: Inter-phase properties

In the case of the "quasi 3-D" single fiber model the algorithm can introduce one, or multiple, debonded zones into the inter-phase layer by using only a few parameters, the debonding extension angle $\alpha$ and its position angle $\theta$ that identifies the center of the injected defect as shown in Fig. 5 for a more complex geometry. The number of parameters increases in the case of complete 3-D models where even the initial position along the fiber direction $z_{0}$ and the depth $t_{z}$ of the decohesion are required.

Moreover in multi-fiber models, in order to reproduce a more realistic presence of defects induced by a manufacturing process, an increased statistical variability of the technique is required. In particular, in this case, it is possible to identify two important variables, the fiber-matrix decohesion density $D_{\%}$ and the decohesions distribution position $D_{P D F}$. The first one, 


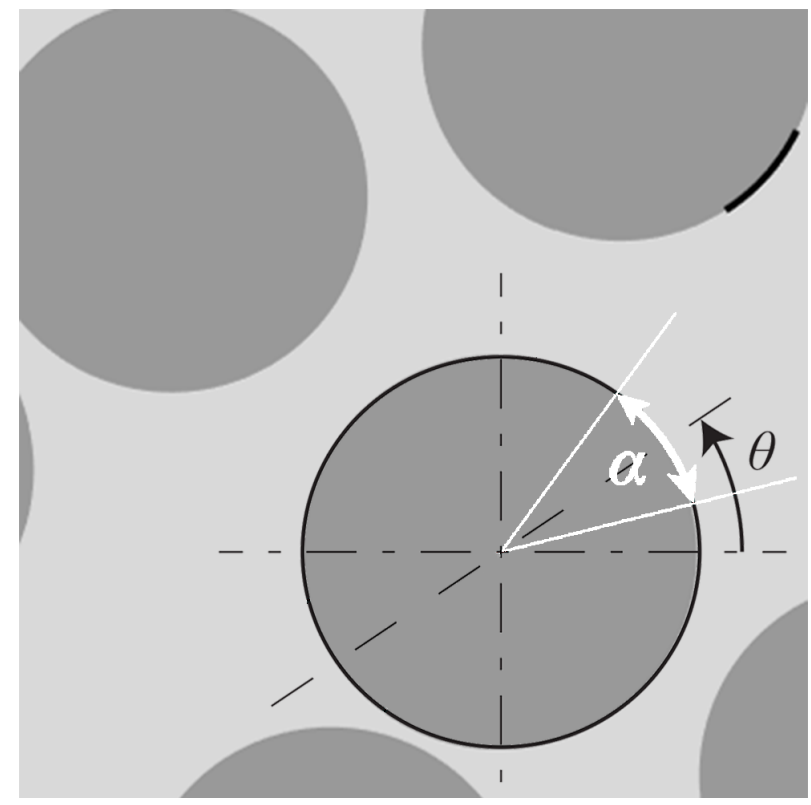

Figure 5: Detail of fiber injection procedure

strictly correlated with the quality of manufacturing process, can be defined as:

$$
D_{\%}=\frac{A_{i_{\text {dam }}}}{A_{i_{T O T}}}
$$

where $A_{i_{\text {dam }}}$ defines the area of inter-phase elements that are damaged while $A_{i_{\text {Tот }}}$ is the total area of inter-phase elements that are present into the model. The other variables introduces a variability component into the models with $D_{P D F}$, in fact it is possible to use different statistical distributions (Uniform, Normal, Weibull, ...) in order to affect both the orientation and the mean size of injected defects taking into account the effect of a possible directionality on defects induced by the process as can be observed in Fig. 6.

The present algorithm in order takes into account for the injection process 


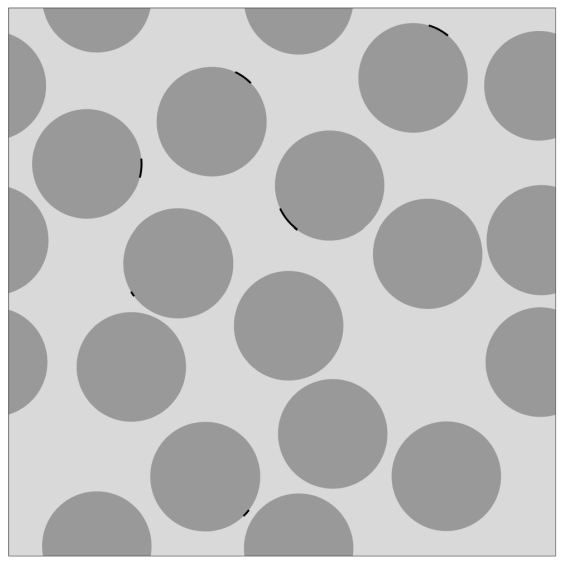

(a) Uniform, $D_{\%}=0.025$

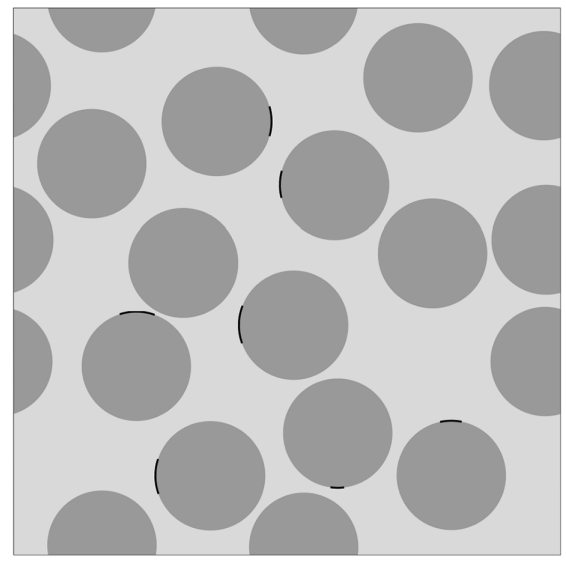

(d) Discrete, $D_{\%}=0.05$

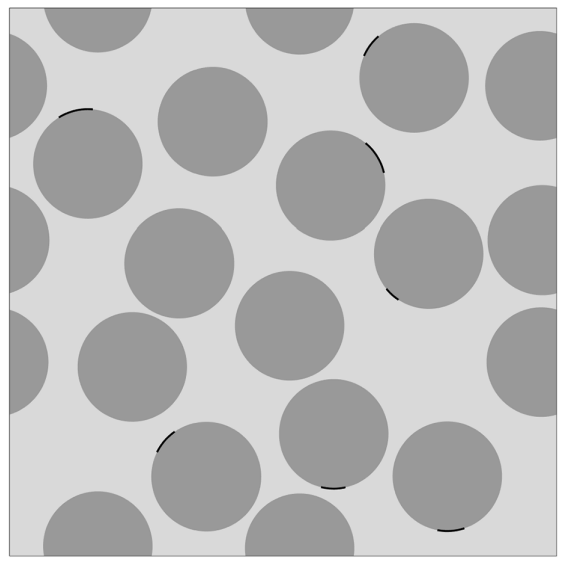

(b) Uniform, $D_{\%}=0.05$ (1)

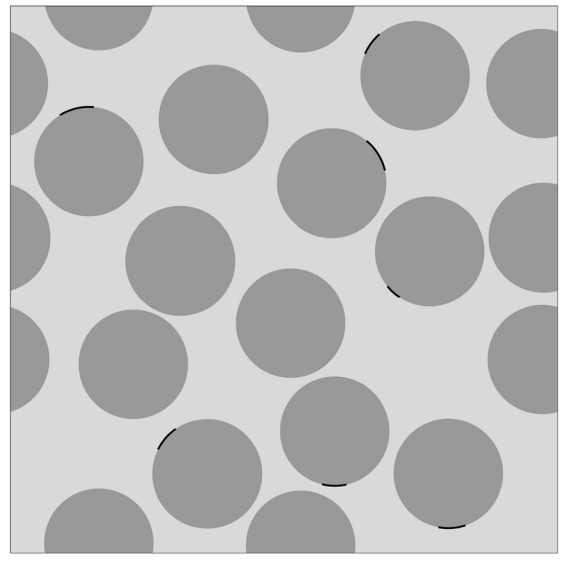

(e) Uniform, $D_{\%}=0.05(2)$

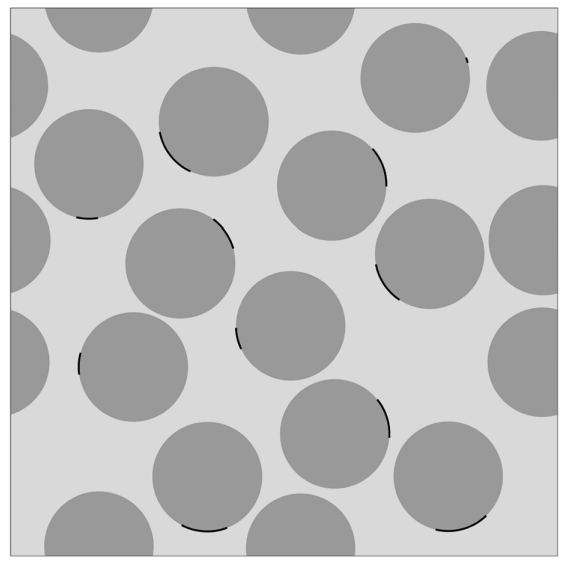

(c) Uniform, $D_{\%}=0.1$

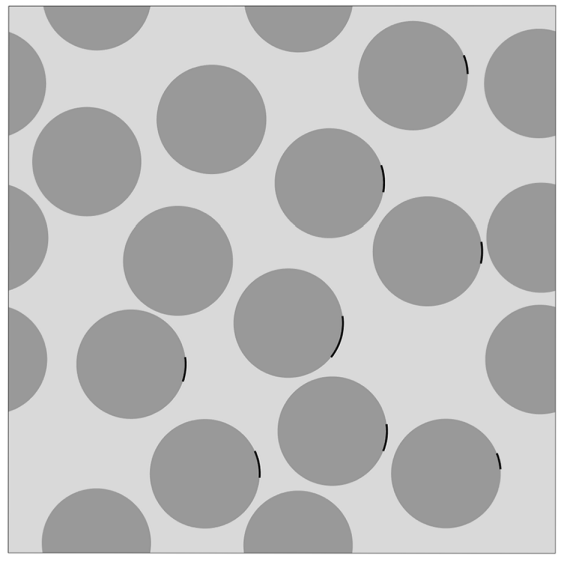

(f) Normal, $D_{\%}=0.05$

Figure 6: Inter-phase damage distributions 
only the fibers that are completely inside the RVE domain in order to not enhance damage localization effect at the boundary of the model. In the RVE model that will be used in the following analyses $D_{\%}$ will be set to 0.05 but in a more general case it is possible to correlate this value with the typical defects density of manufacturing processes.

\section{Technique verification by means of elastic models}

Since the initialization technique has been developed at larger scales for flat geometries, simple single-fiber models are created to be compared with standard FE models in which the fiber is physically detached from the matrix. This preliminary verification, developed into the linear elastic regime, has been done in order to obtain the two-fold objective of verifying this technique in micromechanical models and studying the sensitivity of composite material to flaws in terms of stiffness reduction. For this analyses three different boundary conditions, imposed via PBC, were investigated: transverse tension, pure transverse shear and pure in-plane shear. Defects are introduced as a couple of symmetric flaws with variable amplitude whose position angle is respectively of $\bar{\theta}$ and $\bar{\theta}+180^{\circ}$ in order to preserve models symmetry. $\bar{\theta}=\left[0^{\circ}, 45^{\circ}, 90^{\circ}\right]$ are chosen for transverse tension and plane shear while $\bar{\theta}=\left[45^{\circ}, 90^{\circ}, 135^{\circ}\right]$ are selected for transverse shear case. In order to comprehend how flaws affect the composite material stiffness various relevant extension angle are investigated $\left[15^{\circ}, 30^{\circ}, 45^{\circ}, 60^{\circ}, 90^{\circ}\right]$ and the results compared respect to the pristine model. The comparison between the cohesive injected models and the physical flaw models in terms of stiffness variation for the three considered defects positions are reported in Fig. 7 for each 
loading conditions.

As can be seen from the figure above, the maximum difference among the two approaches in terms of stiffness values is obtained in the plane shear conditions for a defect with $\alpha=45^{\circ}$ and $\theta=0^{\circ}$. Since this difference is lower then $5 \%$ and in the other loading conditions ite is always lower then $1 \%$ it is possible to use the new approach in order to model inter-phase decohesions into more complex micromechanical analyses.

\section{Results for a quasi 3-D single fiber models}

Checked the validity to inject decohesions in models for linear elastic analyses the investigation of composite failure is carried out with several models. In a first instance a series of single fiber models with a single defect of fixed amplitude $\left(\alpha=15^{\circ}\right)$ are created. In order to cover almost all possible debonding positions, the flaw is introduced in a specific position: $\theta=0^{\circ}$ for transverse tension and pure in-plane shear and $\theta=45^{\circ}$ for pure transverse shear; the flaw is moved with a pitch of $10^{\circ}$ up to $90^{\circ}$ for the first two cases and up to $135^{\circ}$ for transverse shear. From these analyses it has been possible to highlight how the two failure mechanisms, fiber-matrix decohesions and matrix plasticity, evolve together till composite failure. The models with a fixed $V_{f}$ of $30 \%$ are obtained with a python script and their size is approximatively of 8000 elements with 7000 C3D8, 150 C3D6 and circa 200 COH3D8 cohesive elements

From the analyses emerged that changes in loading condition strongly affect the influence of fiber-matrix inter-phase into the global composite behavior. In particular, in the case of transverse tension, the presence of an inter-phase 


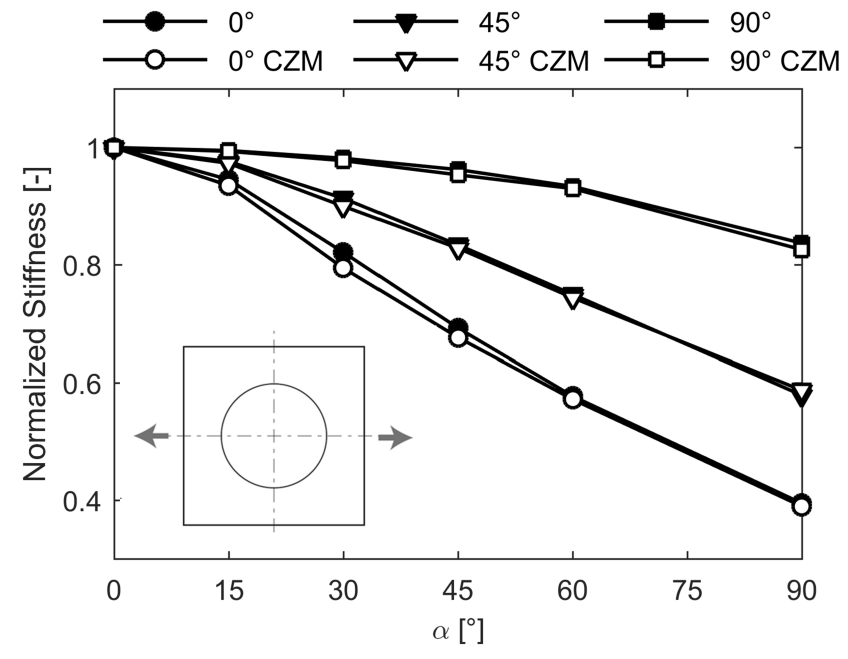

(a) Transverse tension

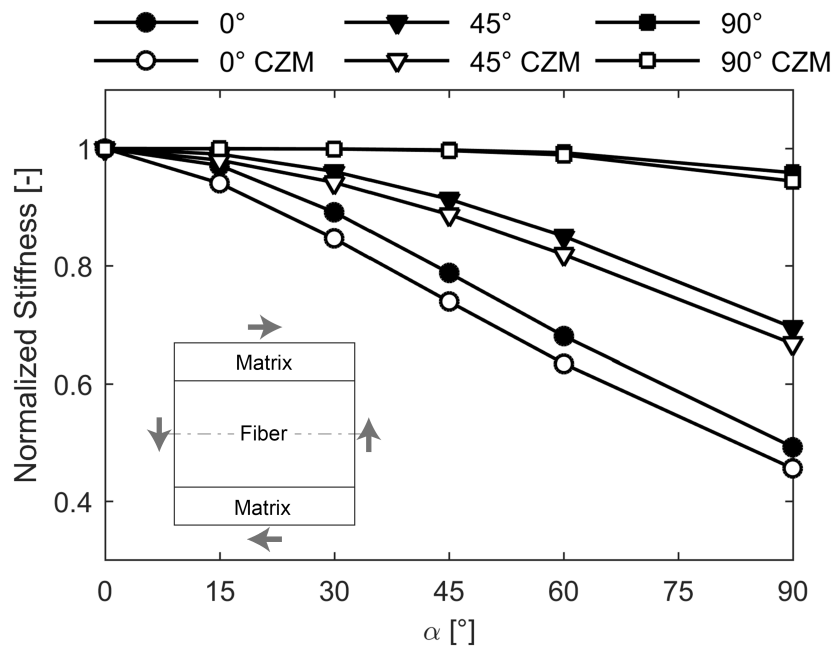

(b) In-plane shear

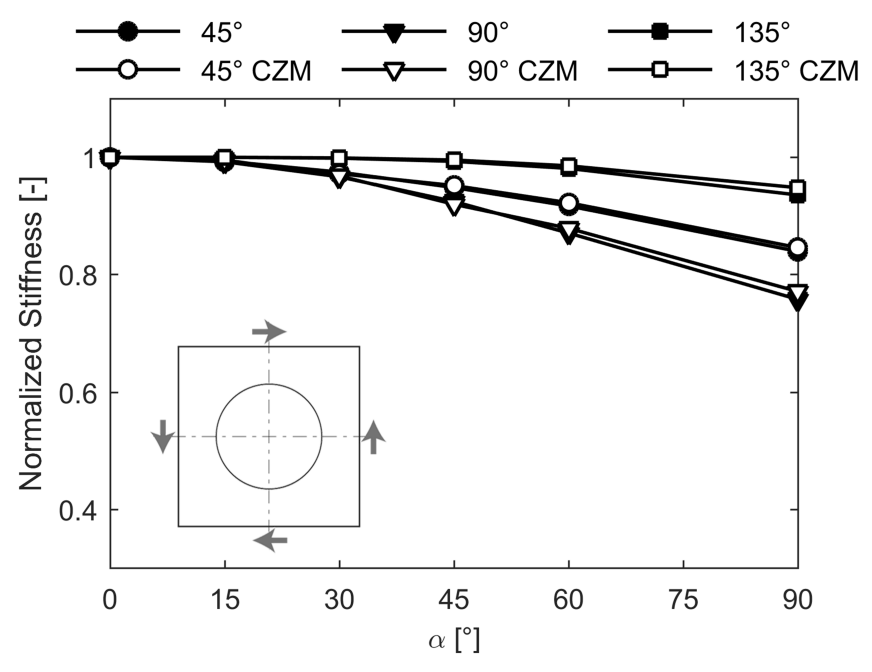

(c) Transverse shear

Figure 7: Stiffness reduction due to inter-phase debonding 
decohesion can reduce the material strength of more than $60 \%$ as reported in 8(a). As it is possible to see from Fig. 9(a), the maximum degradation of the composite mechanical properties appears when the decohesion is positioned at $\theta=10^{\circ}$ because the crack tip is orthogonal with the direction of the greater principal stress while in the case of $\theta>70^{\circ}$ the presence of the flaw is almost irrelevant. An interesting thing to observe is that when $\theta>70^{\circ}$ the inter-phase decohesion does not propagate from the damaged zone but a new crack arise in the zone of the inter-phase that is more stressed in terms opening tension $\left(\theta=10^{\circ}\right)$ as seen in Fig. 9(b).

Plane shear models exhibit a more elasto-plastic behavior and the maximum reduction in terms of strength occurs when the defects is initialized at $\theta=0^{\circ}$ while its effect tends to vanish if $\theta>50^{\circ}$ as shown in Fig. 9(a).

In the last boundary condition all the curves exhibit a large plastic deformation and the failure stress is quite similar for all the position of the flaws. In this case, unlike the previous analyses, it is possible to observe that for small strains a first interphase decohesion occurs and after that large plastic deformation takes place as highlited in Fig. 8(c).

\section{Results for quasi 3-D multifiber RVEs}

The effect of fiber-matrix decohesions injection into a more complex geometrical scenario is reported in this section; in particular the multi-fiber RVE presented in Fig. 6 with a volume fraction of 0.5 are generated and for

each previously investigated loading conditions four analyses are performed at the same damage density $\left(D_{\%}=0.05\right)$. The models are meshed with circa 80000 elements with almost a 90\% of eight node brick (C3D8) elements while 


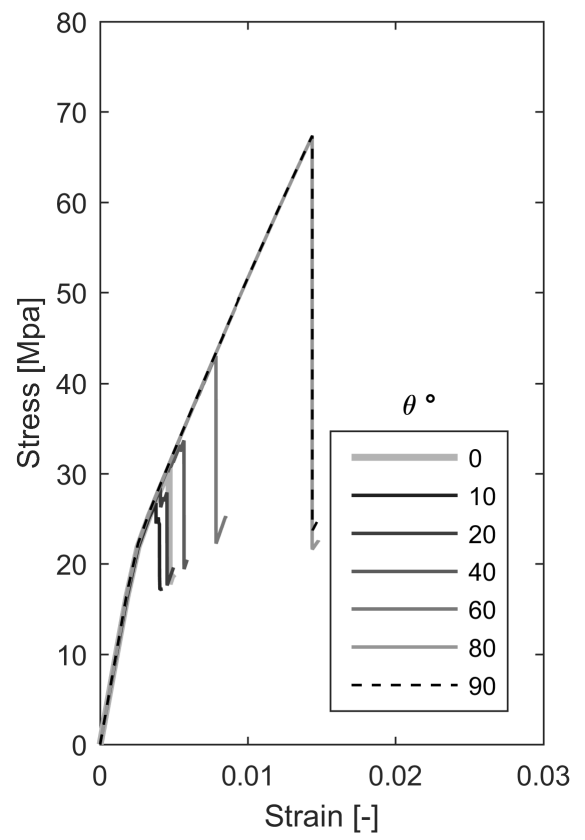

(a) Transverse tension

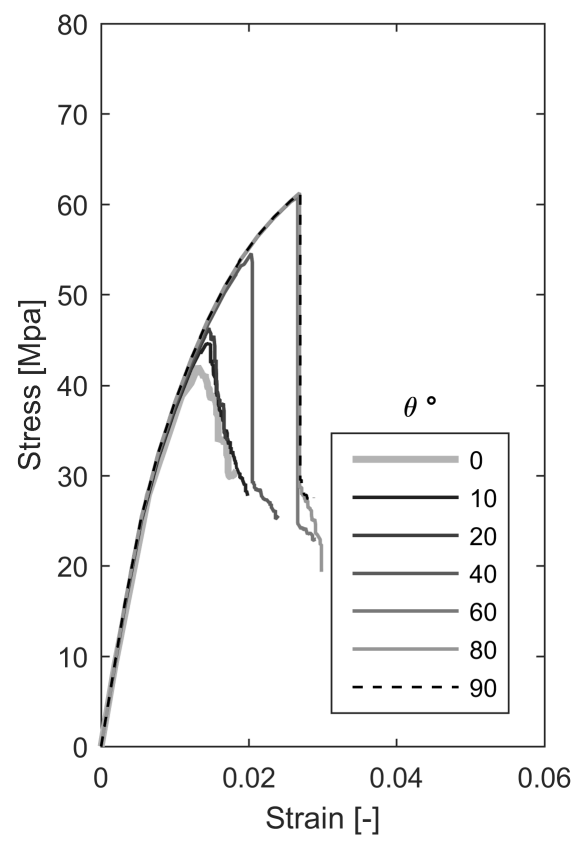

(b) In-plane shear

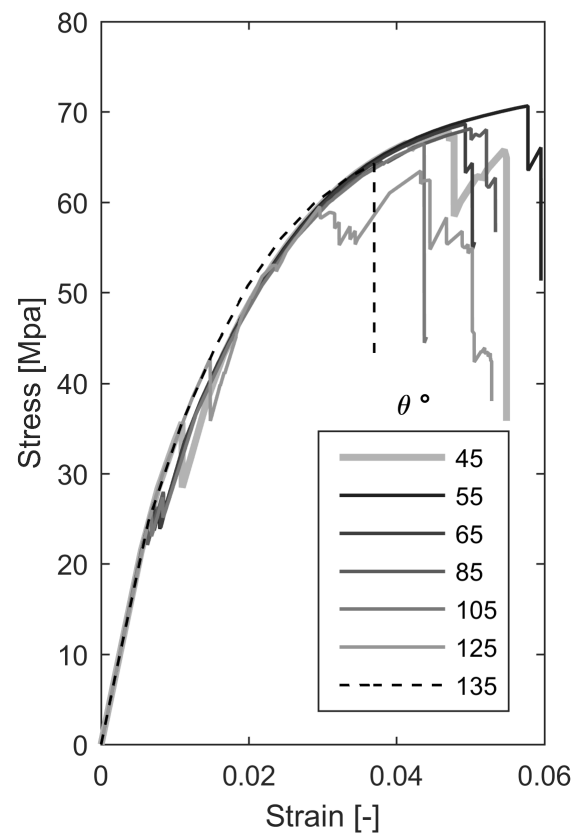

(c) Transverse shear

Figure 8: Stress-strain behavior for different defect positions 


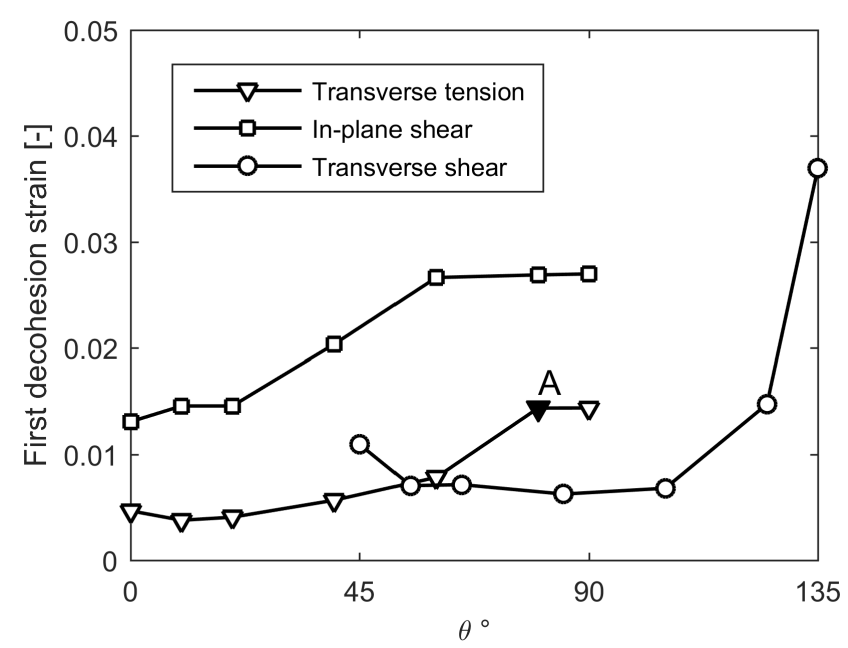

(a) First decohesion strain

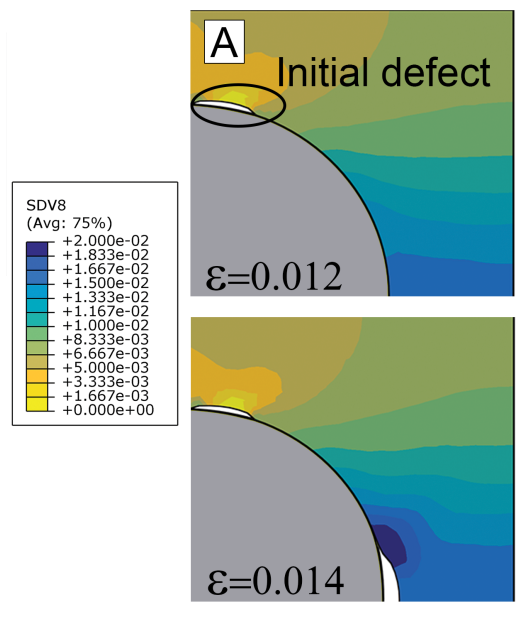

(b) Evolution of interfacial decohesion and of matrix equivalent plastic strain (SDV8)

Figure 9: First interface decohesion 
less then 5\% six node wedge (C3D6) are used as filler and the inter-phase regions are meshed with COH3D8 cohesive elements. The analyses consist of: a first analysis in which the inter-phases are considered all perfectly bonded, a second one with an uniform distribution for the defect opening angle and a normal distribution for the position angle (with the mean value defined as the most effective position, obtained from the previous analyses), and two other models created with defects randomly initialized. Results are compared in terms of stress-strain behavior and crack path evolution.

In the case of transverse tension the analyses highlight that the presence of fiber-matrix injected debonding can strongly affect the onset of mechanical degradation, as reported in Fig. 10(a), with a reduction of the composite strength of $50 \%$.

For Transverse tension condition the quasi 3-D multifiber models have been particularly sensitive to the presence of a crack at $\theta=10^{\circ}$. At the same time models that exhibit a large number of early fiber-matrix debondings (Model-2 and Worst-case) tend to have a more ductile behavior up to failure.

For the in-plane shear PBC the presence of damage into the inter-phase seems not affecting the solution; in fact from the stress-strain curves of Fig. 11(a) is possible to deduce that final failure is dominated by matrix properties. For this loading condition, as shown in Fig. 11(b), the crack path originates from the failure condition of the plasticized matrix and vertically crack propagates up to failure; the decohesion in this case has the only effect of a little anticipation of matrix plasticity and cracking but no evident effect is highlighted. 


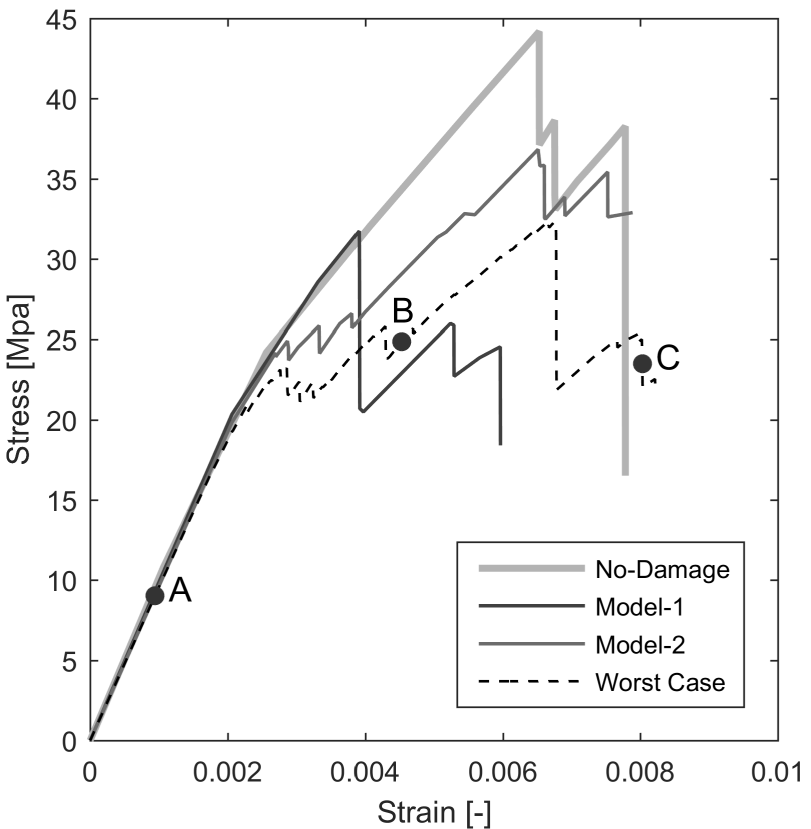

(a) Homogenized stress vs. strain curves
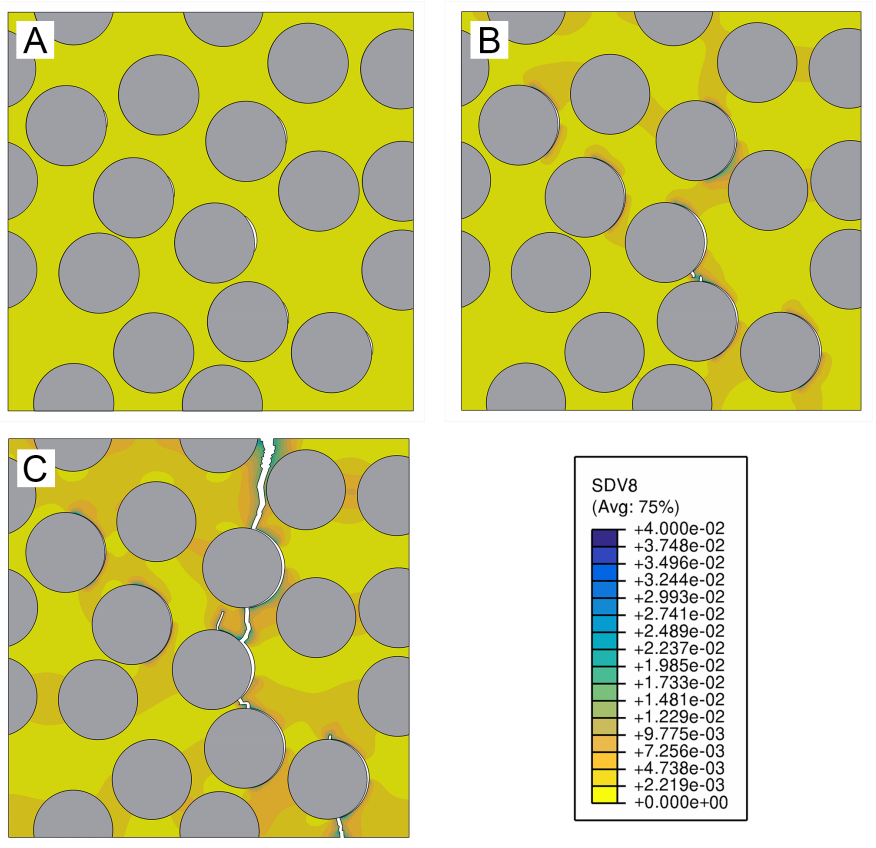

(b) Equivalent plastic strain (SDV8) and damage evolution for worst case analysis

Figure 10: RVE stress-strain behavior in transverse tension 


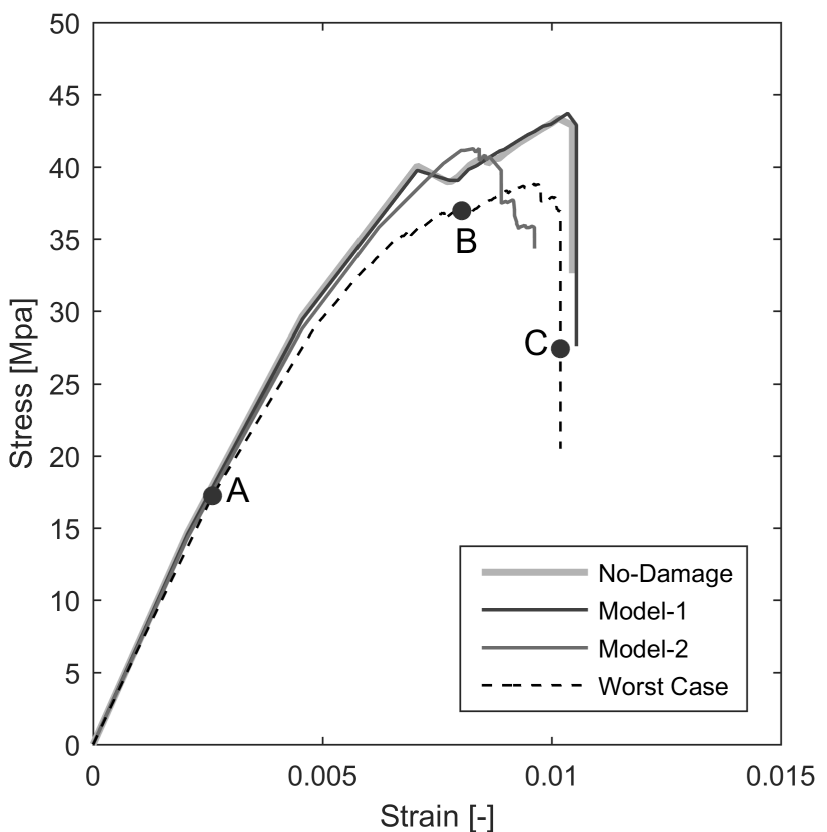

(a) Homogenized stress vs. strain curves
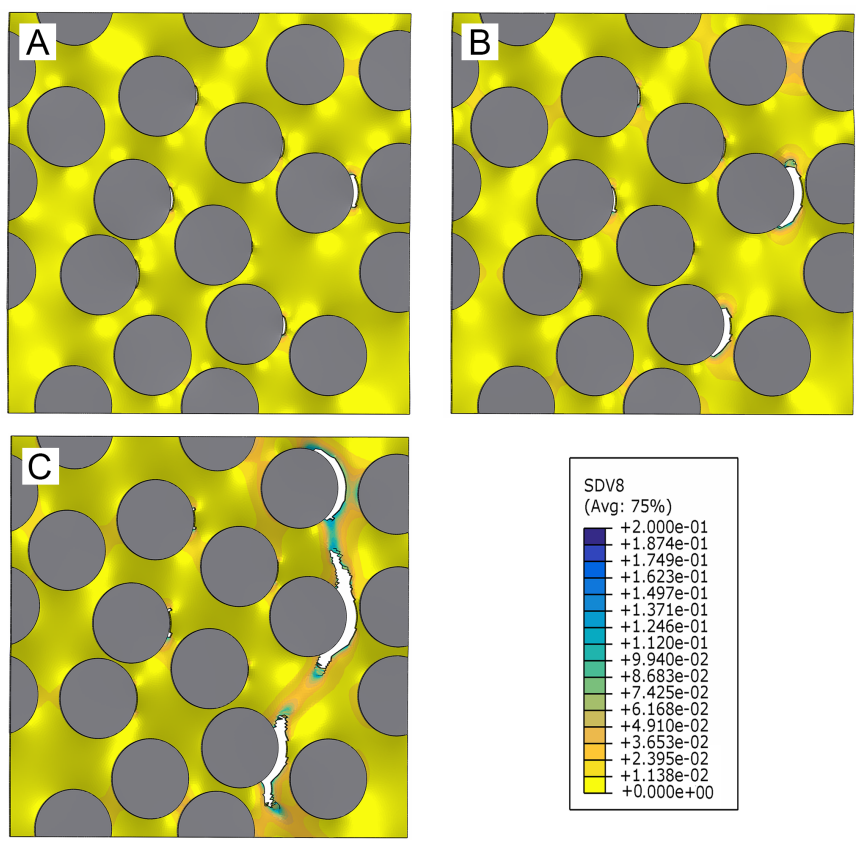

(b) Equivalent plastic strain (SDV8) and damage evolution for worst-case analysis

Figure 11: RVE stress-strain behavior in in-plane shear 


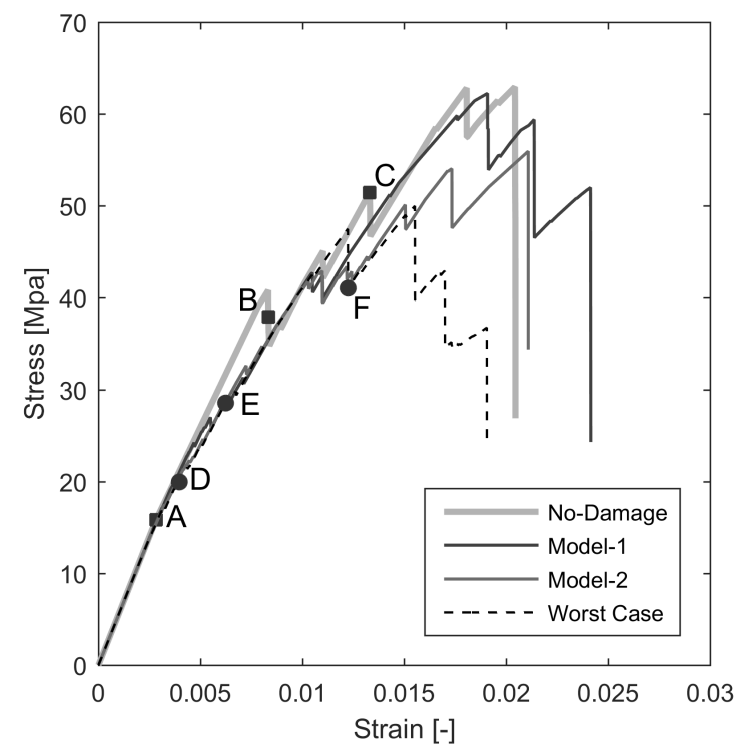

Figure 12: [Homogenized stress vs. strain curves in transverse shear

Under the loading condition of transverse shear the presence of multiple interface defects produces: at small strain, a weak reduction of the mechanical performances while, at higher deformation, it anticipates the material final failure as shown in Fig. 12.

This phenomenon is due to the strong affection of defects onto the damage path propagation in the RVE, as highlighted in Fig. 13. The analyses revealed that, while in the pristine model, as illustrated in Fig. 13(a), matrix cracking appears on a $45^{\circ}$ paths, orthogonal to the first principal stress producing a soft and progressive composite degradation up to the final failure. In the worst-case analysis 13(b) the presence of multiple defects into the most effect position $\left(\theta=90^{\circ}\right)$ induces the crack path growing horizontally with a complete separation of the RVE with the resulting failure 


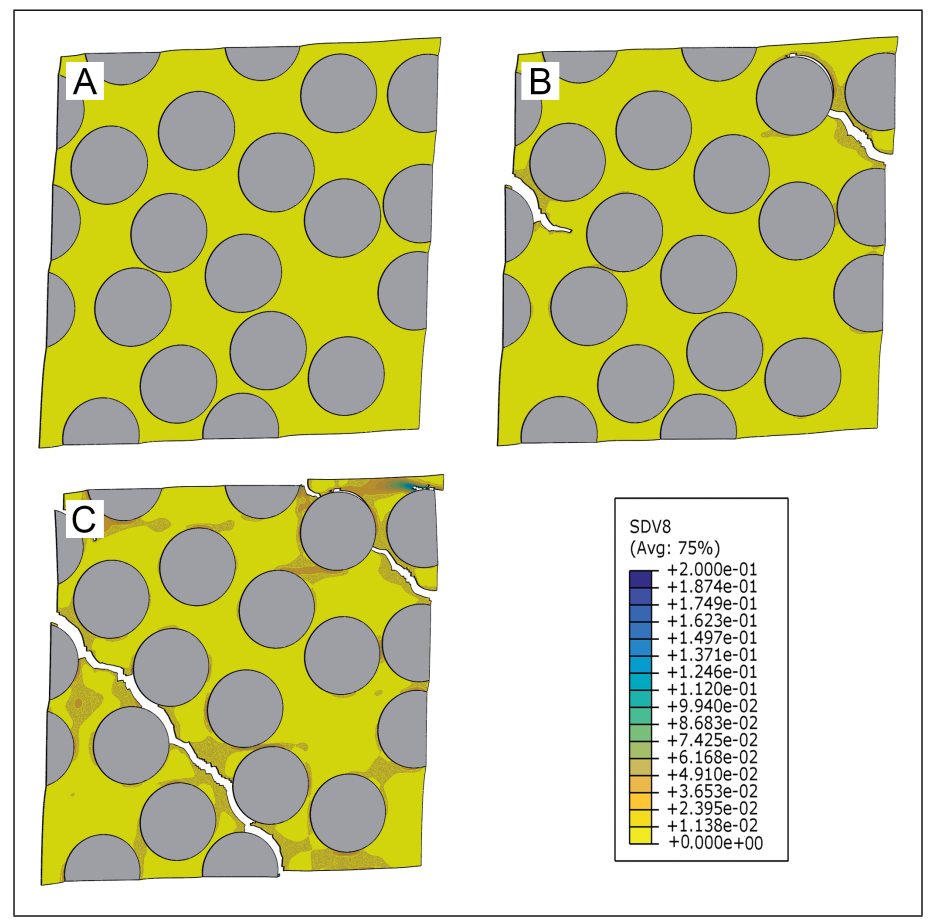

(a) No-damage injected analysis

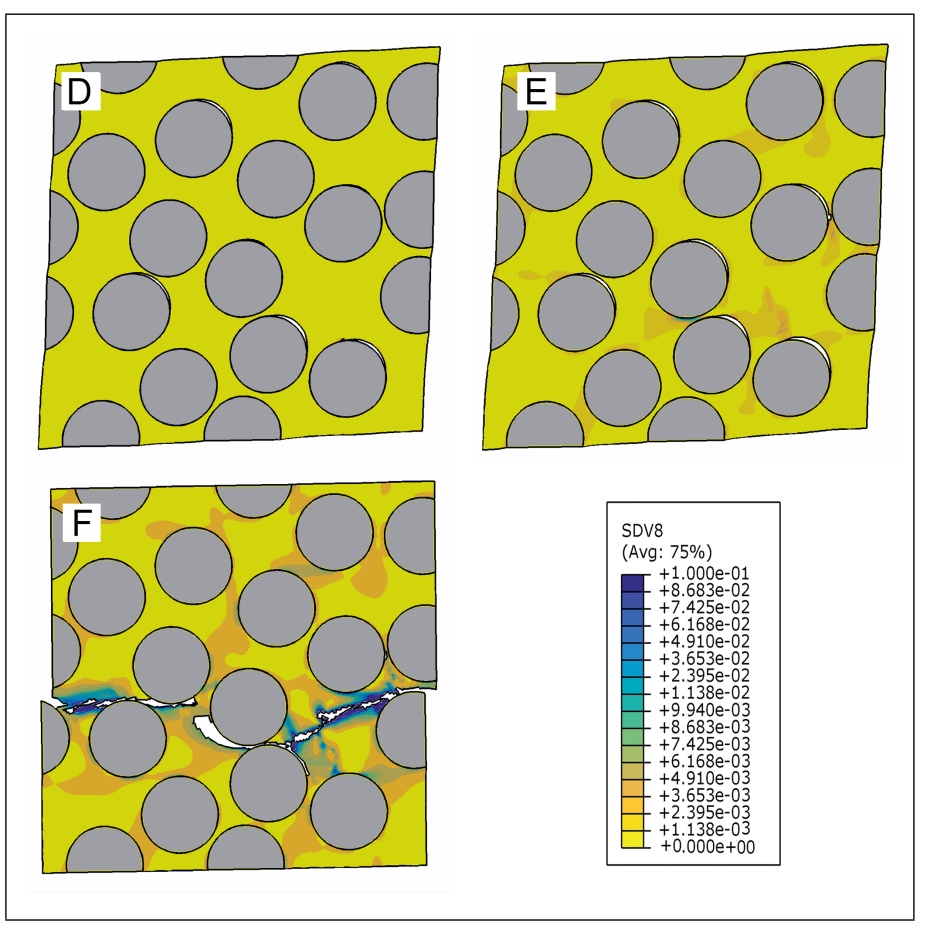

(b) Worst-case defects injection analysis

Figure 13: Equivalent plastic strain (SDV8) and damage evolution 


\section{Results for 3-D single fiber models}

Previous models provided a wide vision of how the presence of fibermatrix debondings can affect the mechanical behavior of unidirectional composites but they are not capable of predicting the propagation of defects along the fiber direction. In order to have a deep insight even into this aspect of inter-phase debonding, models with an increased length along the fiber direction are generated and the defects are injected into the middle of their length. In this preliminary investigation only single fiber models as those seen in Sec. 6 are analyzed and the same boundary condition introduced. Models have been injected with a decohesion angle $\alpha$ of $15^{\circ}$ and an almost square matrix-fiber decohesion is itroduced into the position that was retained most affective from previous analyses. In transverse tension condition the debonding has been injected in the position of $\theta=10^{\circ}$ and the evolution of the flaw till material failure is reported in Fig. 14.

With the application of the load it is possible to observe that the debonding starts propagating both circumferentially and axially producing a circular decohesion; reached a certain angle the flaw stops growing along the circular direction but it continues evolving along the fiber direction. Reached this condition the model tends to have almost the same behavior of the "quasi 3D" case and matrix breaking starts up to final failure. A bit different is the case of in-plane shear as illustrated in Fig. 15. With the application of this boundary condition the crack starts propagating along the fiber direction without circumferential growing and plastic deformation appears around its tips; increasing the load the debonded zone continues the propagation in the same direction till reaches the front face of the model and then appears on 


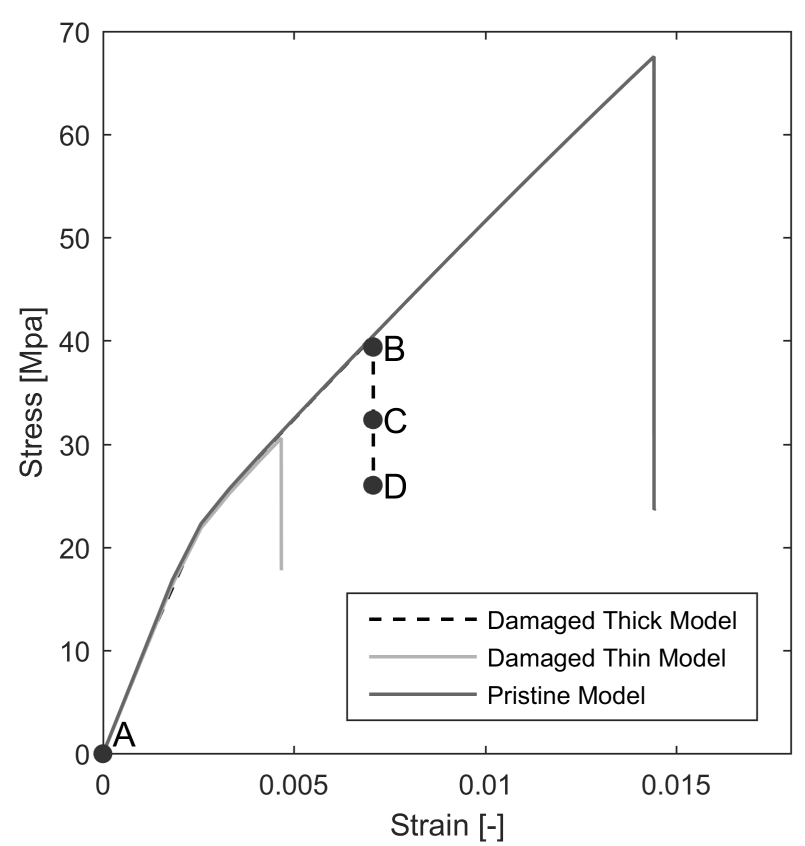

(a) Homogenized stress vs. strain curves

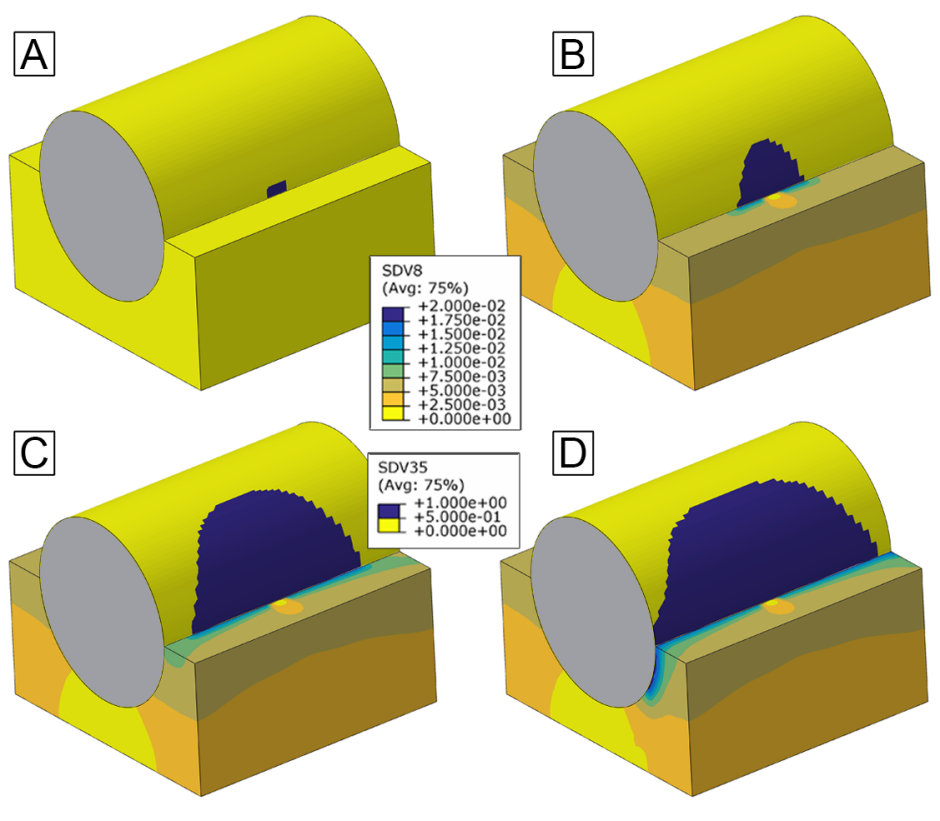

(b) Equivalent plastic strain (SDV8) and evolution of the interfacial damage (SDV35)

Figure 14: Single fiber deep model results in transverse traction 


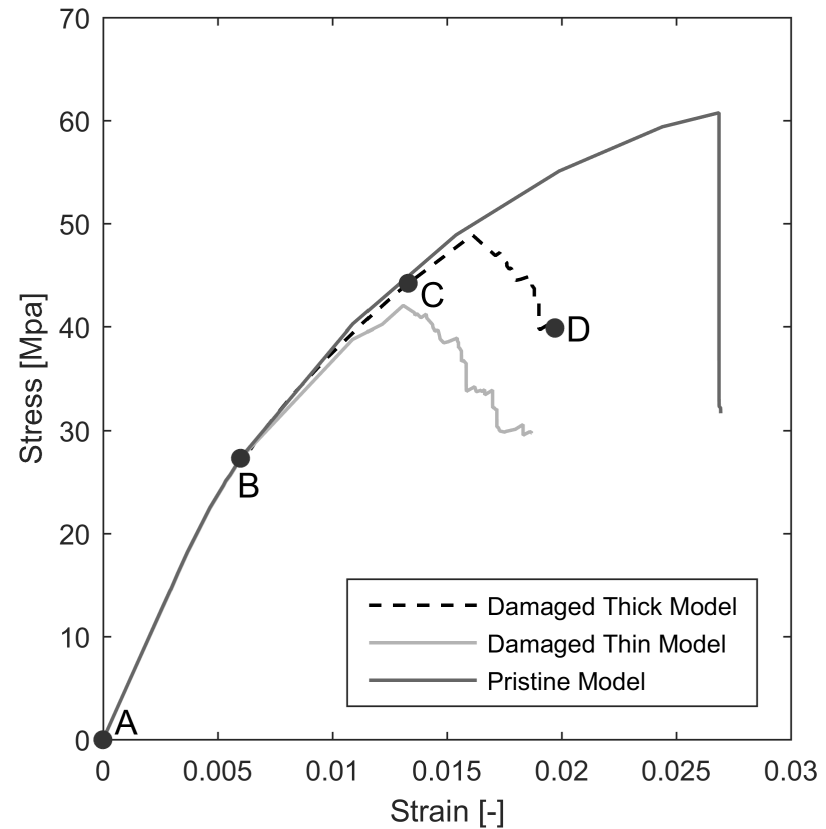

(a) Homogenized stress vs. strain curves

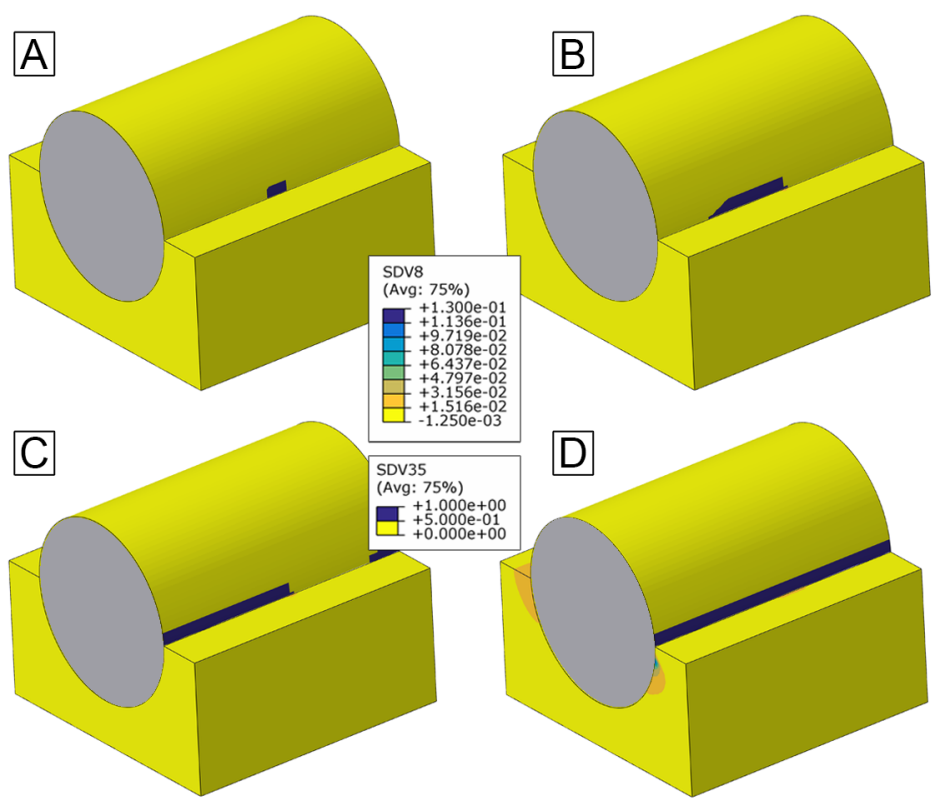

(b) Equivalent plastic strain (SDV8) and evolution of the interfacial damage (SDV35)

Figure 15: Single fiber deep model results in in-plane shear 
its back face. After that the decohesion realizes a through-wall flaws and the plastic zone starts propagatin circumferentially untill the matrix cracking begins. Of particular interest is the case of transverse shear loading in which the inter-phase decohesion is introduced into the most critical position in terms of stiffness reduction $\left(\theta=90^{\circ}\right)$. Applying the $\mathrm{PBC}$ the defect starts propagating almost symmetrically only along the circumferential direction; after this early stage the defect tends to propagate faster towards the side of positive principal stress $\theta=45^{\circ}$. This effect is due to the fact that the principal stress of traction tends to make the decohesion front accelerate while on the other side, $\theta=135^{\circ}$, the compression principal stress do not affect the crack evolution that results slower in propagating. Reached the position of $\theta=45^{\circ}$, the tensioned branch starts propagating both circumferentially and along the fiber direction. At this point, as illustrated, in Fig. 16, the plastic deformation of the matrix manifestly occurs up to the material failure.

\section{Conclusion}

The paper presented the results of a study about the effect of fiber-matrix interphase defects on the mechanical properties of an UD composite. FE analyses have been carried out on micro-scale models in order to asses the influence of the position, extension, shape and number of the interfacial defects.

The study was conducted thanks to the adoption of an original damage injection technique for cohesive elements developed by the authors for meso-scale applications.

This innovative approach to initialize interfacial damage at the micro-scale 


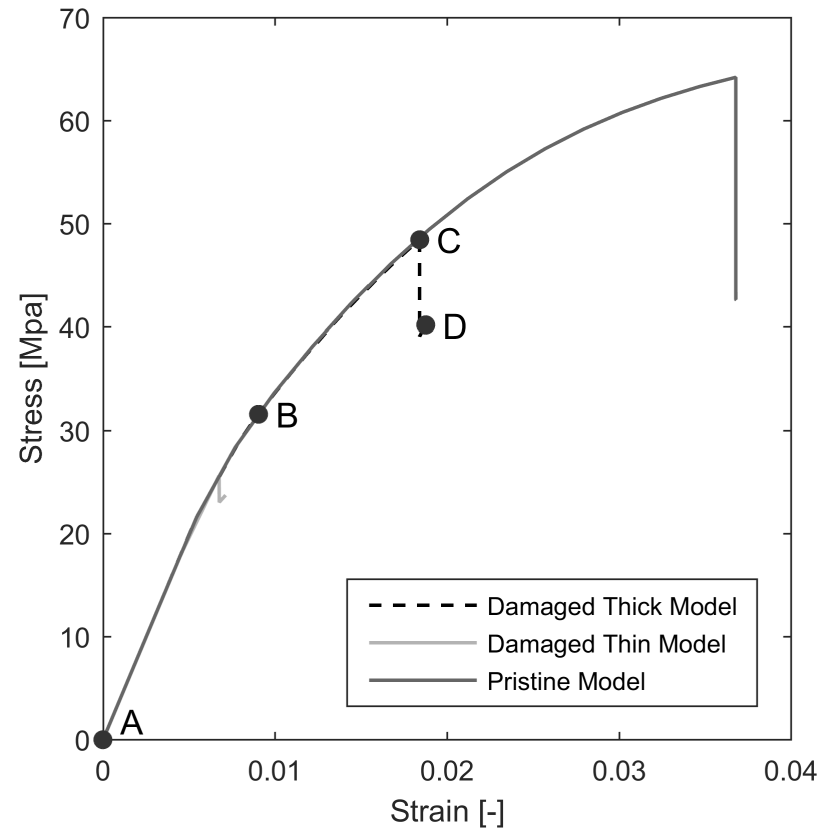

(a) Homogenized stress vs. strain curves
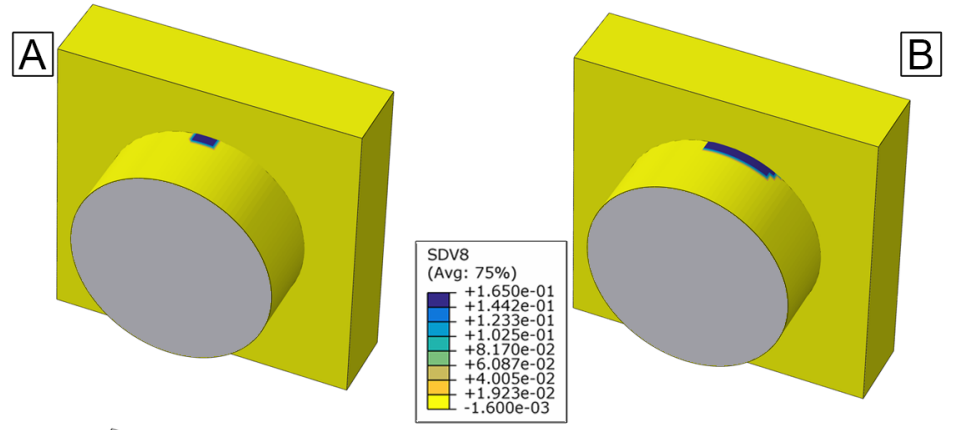

C
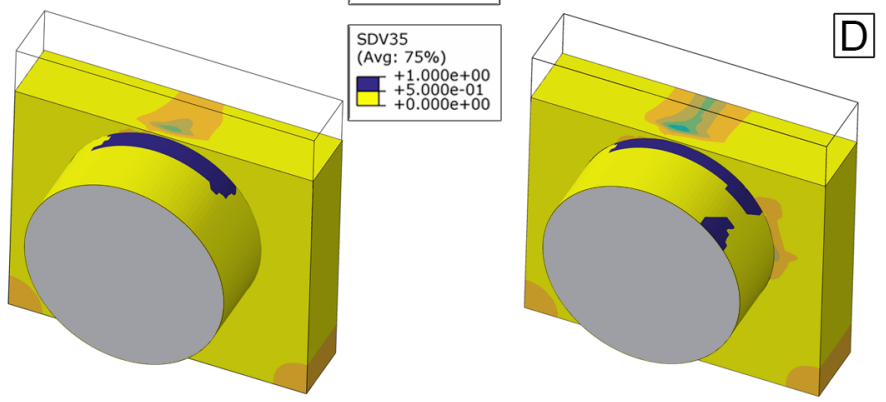

(b) Equivalent plastic strain (SDV8) and evolution of the interfacial damage (SDV35

Figure 16: Single fiber deep model results in transverse shear 
has been verified on single-fiber elastic models. The model with injected fibermatrix de-bonding produced results that closely matched those obtained by means of standard FE models incorporating physical cracks.

The analyses carried out on low-depth single fiber models, with a elastoplastic damageable matrix, showed that an high sensitivity of the results exist to the position of the interfacial decohesion especially for the transverse tension case.

Multi-fibers models confirmed single fiber results highlighting a softening in the stress-strain response that can be attributed mainly to interface and matrix damage for transverse tension while matrix plasticity is the main responsible for the in-plane shear load case; more complex is the transverse shear case where the presence of interfacial decohesion can strongly affect the crack path.

The deep 3-D model allowed studying the propagation of small interface bonding defects The results showed that the defect propagates both circumferentially and axially with different ratios depending on the load case. Generally, the stress-strain response was less affected by the interfacial damage wen compared to its quasi 3-D counterpart thus resulting in a more ductile behavior.

Ultimately, the results presented in the paper contribute to disclose the role played by the presence and the propagation of fiber-matrix interface defects in degrading the stiffness and strength of unidirectional plies of carbon/epoxy composites. 


\section{References}

[1] K.A. Chowdhury, R.R. Talreja, and A.A. Benzerga. Effects of manufacturing-induced voids on local failure in polymer-based composites. ASME. J. Eng. Mater. Technol., 130(2):021010-1-021010-9, 2008.

[2] H. Huang and R. Talreja. Effects of void geometry on elastic properties of unidirectional fiber reinforced composites. Comp. Sci. Tech., 65(13):1964 - 1981, 2005.

[3] L.E. Asp, L.A. Berglund, and R. Talreja. Effects of fiber and interphase on matrix-initiated transverse failure in polymer composites. Composites Science and Technology, 56(6):657-665, 1996. cited By 47.

[4] P. Wriggers, G. Zavarise, and T.I. Zohdi. A computational study of interfacial debonding damage in fibrous composite materials. Computational Materials Science, 12(1):39 - 56, 1998.

[5] J. Segurado and J. Llorca. A computational micromechanics study of the effect of interface decohesion on the mechanical behavior of composites. Acta Materialia, 53(18):4931-4942, 2005. cited By 75.

[6] E. Totry, J.M. Molina-Aldaregua, C. Gonzlez, and J. LLorca. Effect of fiber, matrix and interface properties on the in-plane shear deformation of carbon-fiber reinforced composites. Composites Science and Technology, 70(6):970-980, 2010. cited By 77.

[7] A.R. Maligno, N.A. Warrior, and A.C. Long. Effects of interphase material properties in unidirectional fibre reinforced composites. Composites Science and Technology, 70(1):36-44, 2010. cited By 36. 
[8] J. Llorca, C. Gonzlez, J.M. Molina-Aldaregua, J. Segurado, R. Seltzer, F. Sket, M. Rodrguez, S. Sdaba, R. Muoz, and L.P. Canal. Multiscale modeling of composite materials: A roadmap towards virtual testing. Advanced Materials, 23(44):5130-5147, 2011. cited By 68.

[9] I.M. Gitman, H. Askes, and L.J. Sluys. Representative volume: Existence and size determination. Engineering Fracture Mechanics, 74(16):2518 - 2534, 2007.

[10] L. Yang, Y. Yan, Z. Ran, and Y. Liu. A new method for generating random fibre distributions for fibre reinforced composites. Composites Science and Technology, 76:14-20, 2013.

[11] A.R. Melro, P.P. Camanho, F.M. Andrade Pires, and S.T. Pinho. Micromechanical analysis of polymer composites reinforced by unidirectional fibres: Part i constitutive modelling. International Journal of Solids and Structures, 50(1112):1897 - 1905, 2013.

[12] B. Fiedler, M. Hojo, S. Ochiai, K. Schulte, and M. Ando. Failure behavior of an epoxy matrix under different kinds of static loading. Composites Science and Technology, 61(11):1615 - 1624, 2001.

[13] J. Varna, L.A. Berglund, and M.L. Ericson. Transverse single-fibre test for interfacial debonding in composites: 2. modelling. Comp. Part A, 28(4):317 - 326, 1997.

[14] W. Wang, K. Sadeghipour, and G. Baran. Finite element analysis of the effect of an interphase on toughening of a particle-reinforced polymer 
composite. Composites Part A: Applied Science and Manufacturing, 39(6):956-964, 2008. cited By 21.

[15] A.R. Melro, P.P. Camanho, F.M. Andrade Pires, and S.T. Pinho. Micromechanical analysis of polymer composites reinforced by unidirectional fibres: Part $\{\mathrm{II}\}$ micromechanical analyses. International Journal of Solids and Structures, 50(1112):1906 - 1915, 2013.

[16] D.J. O'Dwyer, N.P. O'Dowd, and C.T. McCarthy. Numerical micromechanical investigation of interfacial strength parameters in a carbon fibre composite material. J. Compos. Mater., 48(6):749 - 760, 2014.

[17] E. Panettieri, D. Fanteria, and A. Firrincieli. Damage initialization techniques for non-sequential fe propagation analysis of delaminations in composite aerospace structures. Meccanica, 50(10):2569-2585, 2015.

[18] Simulia Corp. Abaqus 6.14 User's Manual. Dassault Systèmes Simulia Corp., Providence, RI, USA., 2014.

[19] E.J. Barbero. Finite Element Analysis of Composite Materials. Taylor \& Francis, 2007.

[20] P.D. Soden, M.J. Hinton, and A.S. Kaddour. Lamina properties, layup configurations and loading conditions for a range of fibre-reinforced composite laminates. Comp. Sci. Tech., 58(7):1011 - 1022, 1998.

[21] J.C. Simo and J.W. Ju. Strain- and stress-based continuum damage modelsi. formulation. Int. Jour. Sol. Stru., 23(7):821 - 840, 1987. 
[22] Raghava R., Caddell R.M., and Yeh G.S.Y. The macroscopic yield behaviour of polymers. Jour. Mat. Sci., 8(2):225-232, 1973.

[23] Z. P. Bažant. Mechanics of distributed cracking. Applied Mechanics Reviews, 39:675-705, 1986.

[24] A. Turon, P.P. Camanho, J. Costa, and C.G. Dávila. A damage model for the simulation of delamination in advanced composites under variable-mode loading. Mech. Mat., 38(11):1072 - 1089, 2006.

[25] M.L. Benzeggagh and M. Kenane. Measurement of mixed-mode delamination fracture toughness of unidirectional glass/epoxy composites with mixed-mode bending apparatus. Composites Science and Technology, 56(4):439 - 449, 1996.

[26] T.J. Vaughan and C.T. McCarthy. Micromechanical modelling of the transverse damage behaviour in fibre reinforced composites. Composites Science and Technology, 71(3):388 - 396, 2011. 